\title{
招待講演
}

\section{Efficacy of the Accent Method of Voice Therapy.}

\section{M.Nasser Kotby,M.D., M.Ch.}

Department of Otolaryngology and Unit of Phoniatrics, Faculty of Medicine, Ain Shams University, Cairo, Egypt

There is an increasing need for studies on efficacy of behavior re-adjustment therapy procedures in human communicative disorders. Legal, social, scientific and professional considerations point up the need for more careful documentation of the effects of treatment techniques used by Phoniatricians and Speech-language Pathologists. This study is conducted in order to evaluate the efficacy of the Accent Method of Voice Therapy (AM). The AM is one of the holistic approaches for behavior re-adjustment voice therapy. It tackles collectively and simultaneously the various parameters of voice as pitch, loudness and timbre. The results of intervention utilizing the AM in this clinical trial are assessed in a relatively controlied set up. Patients with voice problems resulting from various etiologic vocal pathologies are distributed randomly into 2 groups. Group $I$ (G-I) is given the full aspect of the AM, that is, voice hygiene advice plus the Accent exercises, to correct the faulty vocal technique (habit). Group II (GII) receives only voice hygiene advice. The AM is administered in individual sessions 20 minutes each, twice per week, while the voice hygiene advice counseling is given once per week. The assessment of the vocal pathology is done following a diagnostic protocol utilizing subjective as well as quasi objective measures of evaluation. The initial assessment presents the base line (pre-test) data for both groups. Two follow up evaluations are done at mid intervention (midtest), that is, 10 sessions for (G-I) and 5 sessions for (G-II), and at the termination of intervention/therapy (post-test). The difference in improvement between G-I and G-II at the end of the observation was generally significant in favour of G-I. There were significant improvements in G-I in certain items specific for the various etiologic categories. The improvement from pretest to mid test to post test values followed a linear tendency. The significance of the results is discussed and the conclusions are outlined and criticized. 